\title{
Cotargeting EGFR and autophagy signaling: A novel therapeutic strategy for non-small-cell lung cancer (Review)
}

\author{
XINBING SUI $^{1 *}$, NA KONG $^{1 *}$, MINGHUA ZHU ${ }^{2}$, XIAN WANG $^{1}$, FANG LOU $^{1}$, \\ WEIDONG HAN ${ }^{1,3}$ and HONGMING PAN ${ }^{1,3}$ \\ ${ }^{1}$ Department of Medical Oncology, Sir Run Run Shaw Hospital, Zhejiang University, Hangshou, Zhejiang;
${ }^{2}$ Department of Nephrology, Hebei Medical University Affiliated North China Petroleum Bureau General Hospital, Renqiu,
Hebei; ${ }^{3}$ Biomedical Research Center and Key Laboratory of Biotherapy of Zhejiang Province, Hangzhou, Zhejiang, P.R. China
}

Received March 17, 2013; Accepted September 9, 2013

DOI: $10.3892 / \mathrm{mco} .2013 .187$

\begin{abstract}
Epidermal growth factor receptor (EGFR) somatic mutations are found in the majority of non-small-cell lung cancers (NSCLCs) and patients with NSCLC who harbor EGFR mutations have been shown to exhibit increased sensitivity to the small-molecule EGFR-tyrosine kinase inhibitors (TKIs) gefitinib and erlotinib. However, the majority of tumors develop acquired resistance to EGFR-TKIs after a median of 10-16 months, which limits the clinical efficacy of these drugs. Autophagy, an important homeostatic cellular recycling mechanism, has emerged as a potential target for the acquired resistance phenotype. Recently, several studies demonstrated that autophagy may be induced in a dose-dependent manner by treatment of multiple cancer cell lines with EGFR-TKIs in vitro. Furthermore, it was recently reported that autophagy, as a cytoprotective response, may be activated by EGFR-TKIs in lung cancer cells and that the inhibition of autophagy enhanced the cytotoxic effect of EGFR-TKIs. In this review, we aimed to focus on the association between resistance to EGFR-TKIs and autophagy, and assess whether autophagy inhibition represents a promising approach to improve the efficacy of EGFR-TKIs in the treatment of NSCLC patients.
\end{abstract}

Correspondence to: Professor Hongming Pan or Dr Weidong Han, Department of Medical Oncology, Sir Run Run Shaw Hospital, Zhejiang University, 3 Qingchun East Road, Hangzhou, Zhejiang 310016, P.R. China

E-mail: drpanhm@aliyun.com

E-mail: weidongh@yahoo.com

*Contributed equally

Key words: epidermal growth factor receptor, autophagy, non-small-cell lung cancer, resistance

\section{Contents}

1. Introduction

2. EGFR and autophagy regulation

3. Autophagy inhibition may be a promising therapeutic strategy for enhancing the effects of EGFR-targeted therapy in NSCLC

4. Issues and perspectives

\section{Introduction}

Non-small-cell lung cancer (NSCLC) remains the leading cause of cancer-related mortality worldwide (1). NSCLC is closely associated with mutations of the epidermal growth factor receptor (EGFR), which are associated with adenocarcinoma histology, female gender, Asian ethnicity and a non-smoker status $(2,3)$. Subsequent to ligand binding, EGFR becomes activated via phosphorylation. Phosphorylated EGFR subsequently activates pro-survival and antiapoptotic signals through its downstream targets, such as Ras/Raf/MEK/MAPK, JAK/STAT, PI3K/AKT/mTOR, NCK/PAK/JNK and PLC/PAG/PKC (Fig. 1) (4,5). Consequently, inhibition of the EGFR network may activate the intrinsic mitochondrial apoptotic pathway and exhibit antitumor activity against NSCLC. Two specific EGFR-tyrosine kinase inhibitors (TKIs), gefitinib and erlotinib, have been approved by the Food and Drug Administration (FDA) thus far for the treatment of patients with advanced NSCLC. Notwithstanding the success of these drugs in cases of NSCLC with classical EGFR mutations, it appears that all the cases ultimately relapse, resulting in acquired resistance to EGFR-TKIs (6). These unsatisfactory outcomes are, in part, due to macroautophagy (hereafter autophagy), which is considered to be crucial in the resistance to EGFR-TKIs.

Autophagy is a catabolic process in which portions of cytosol and organelles are sequestered into a double-membrane vesicle and delivered to the lysosome for bulk degradation to maintain cellular homeostasis $(7,8)$. The role of autophagy in regulating cancer cell death or survival has not been fully elucidated. When cancer cells are subjected to unfavorable conditions, such as nutrient-deficient environment or treatment 


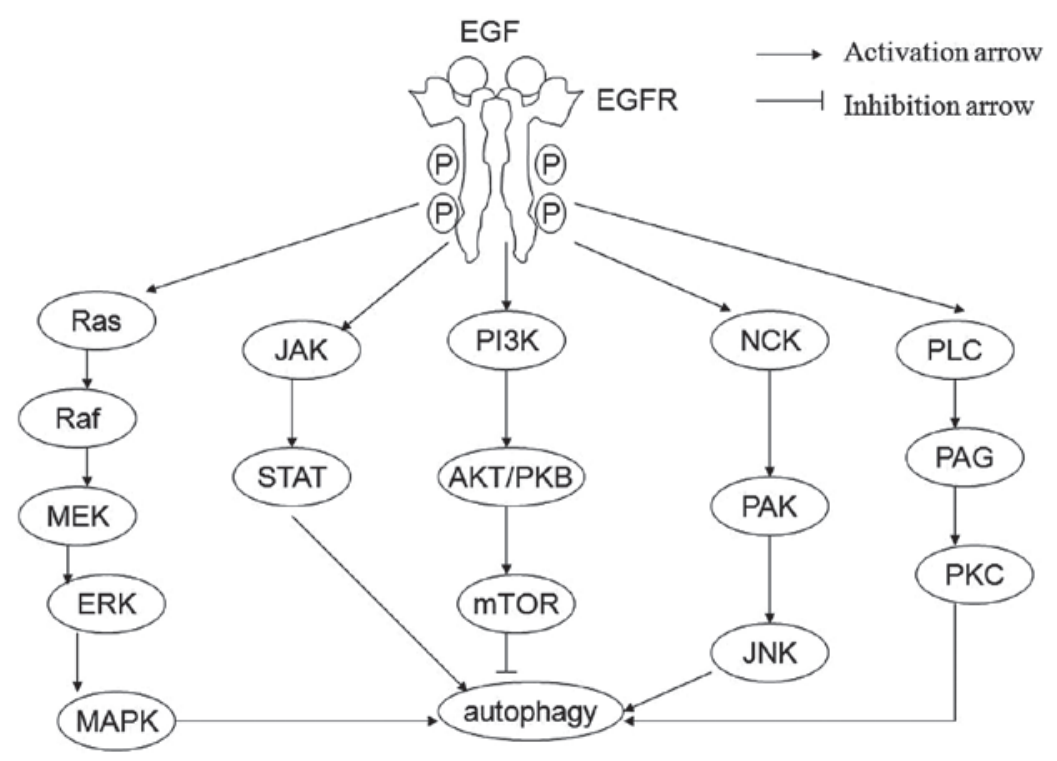

Figure 1. Plasma membrane-bound epidermal growth factor receptor (EGFR) and autophagy signaling network. Following ligand binding, EGFR is activated via phosphorylation. Phosphorylated EGFR subsequently activates the intrinsic tyrosine kinase activity of the receptor and triggers downstream signaling cascades. The major downstream pathways of EGFR include Ras/Raf/MEK/ERK/MAPK, JAK/STAT, PI3K/AKT/mTOR, NCK-PAK-JNK and PLC-PAG-PKC. All these signals exert a strong stimulatory effect on autophagy.

with anticancer drugs, autophagy is rapidly upregulated to function as a cytoprotective mechanism which enhances cancer cell survival, and may contribute to the resistance to anticancer therapies (9). Excessive autophagy may also lead to autophagic cell death, which is also referred to as type II programmed cell death to distinguish it from type I programmed cell death (apoptosis) (10). Various stress signals may induce autophagy,including p53, phosphatidylinositol 3-kinase (PI3K)/mammalian target of rapamycin (mTOR), AMP-activated protein kinase (AMPK) and other signaling pathways $(11,12)$. Among these signals, the AMPK/mTOR axis is critical for the regulation of autophagy. Since PI3K/AKT/mTOR signaling is involved in the regulation of EGFR as well as in autophagic flux, there is likely an association between autophagy and EGFR. Our laboratory recently reported that EGFR-TKIs may activate cell autophagy to impair the sensitivity of lung cancer cells to targeted therapy by inhibiting the AKT/mTOR/p70S6K signaling pathway (13). In this review, we aimed to summarize recent studies on the ability of EGFR-TKIs to induce autophagy, assess whether this ability is correlated with EGFR-targeted therapy resistance and investigate the prospect of manipulating this ability as a novel method of sensitizing cancer cells to these type of drugs.

\section{EGFR and autophagy regulation}

The EGFR signaling pathway is frequently dysregulated in a variety of human malignancies, which has attracted interest in EGFR as a target of anticancer therapies. Several small-molecule TKIs, such as erlotinib or gefitinib, as well as anti-EGFR antibodies, such as cetuximab, have been approved by the FDA and the European Medicines Evaluation Agency for the treatment of patients with breast and colorectal cancer, NSCLC, squamous cell carcinoma of the head and neck and pancreatic cancer (14-16).

Although generally known as a plasma membrane protein, EGFR has also been identified in the nucleus and mitochondria $(17,18)$. Increasing evidence has indicated that the EGFR functionality may be dependent on its subcellular location. In the plasma, EGFR is best known for its classical function as a membrane-bound receptor tyrosine kinase, which is phosphorylated following ligand binding to activate its downstream targets, which are involved in the regulation of autophagy. However, in the nucleus, EGFR behaves as a transcriptional regulator and mediator of other physiological processes. There is currrently no evidence to elucidate whether EGFR is associated with autophagy. Recently, the mitochondrial localization of EGFR was also reported, although the role of mitochondrially localized EGFR is unknown (19). A novel role of mitochondrial EGFR, that appears to regulate apoptosis and autophagy, was recently identified (Fig. 2) (19,20).

Cytoplasmic EGFR is a member of the human epidermal growth factor receptor (HER)/ErbB family of receptor tyrosine kinases. Upon binding to its ligand, EGFR undergoes homoor heterodimerization with its family members ErbB2/neu, ErbB3/HER3 and ErbB4/HER4, which leads to the phosphorylation of EGFR and other intracellular signaling proteins and subsequently initiates a number of downstream signaling molecules that are critical for cancer cell growth, survival and proliferation $(21,22)$. The main pathways triggered by EGFR activation include MAPK, JAK/STAT, PI3K/AKT/mTOR, NCK/PAK/JNK and PLC/PAG/PKC, all of which exert a potent stimulatory effect on autophagy (Fig. 1) (4,5). Although autophagy enhanced by troglitazone is considered to be independent of EGFR transactivation, it was more recently demonstrated that EGFR may mediate autophagy through its downstream signals, such as the AMPK/mTOR pathway (23). Mammary epithelial cells expressing the ${ }^{\text {delE746-A750 EGFR onco- }}$ gene have a functional AMP/LKB1/AMPK $\alpha$ sensor circuitry. Moreover, EGFR activation protects against ultraviolet-induced apoptosis in cultured mouse dendritic cells through the induction of the LKB1/AMPK pathway $(24,25)$. The EGFR inhibitor BIBU may induce apoptosis and prevent execution of autophagy 


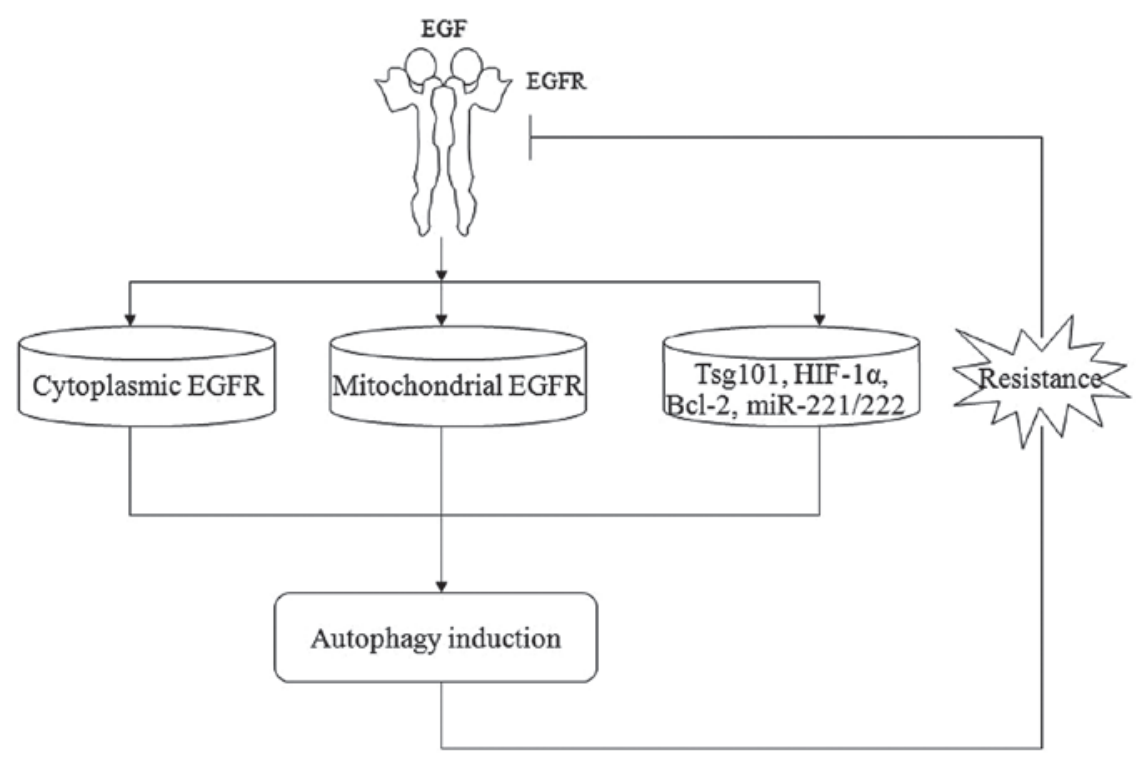

Figure 2. Association between autophagy and acquired epidermal growth factor receptor-tyrosine kinase inhibitors (EGFR-TKIs) resistance. Cytoplasmic EGFR contributes to autophagic regulation through its downstream signaling and EGFR mitochondrial translocation modulated by autophagy may be critical for cell survival in certain types of cancer cells. Furthermore, tumor susceptibility gene 101 (Tsg101), HIF-1 $\alpha$, Bcl-2 and miR-221/222 are also involved in the regulation of EGFR and autophagy. All these factors may contribute to the resistance to EGFR-TKIs in patients with non-small cell lung cancer.

in glioma cells via the inhibition of the pro-survival pathways AKT/mTOR and gp130/JAK/STAT3 (26). The MAPK pathway may transmit signals from the receptor to the nucleus through a series of intermediate proteins, including Ras, Raf, MEK and ERK. Akhtar et al (27) reported that activation of EGFR via pathways involving ERK1/2, p38 MAPK and AKT/FOXO promoted cardiac cell survival during ischemia-reperfusion injury. Chen et al (28) demonstrated that the surface receptor EGFR and its downstream ERK/MAPK signaling pathway are crucial in the activation of autophagy in cathepsin S-targeted cells. Furthermore, a recent study indicated that EGFR downstream signal JNK exerts a major protective effect by increasing autophagy, leading to antagonising apoptosis and ROS generation in A431 cells (29). These data support the fact that cytoplasmic EGFR contributes to autophagic regulation by its downstream signalings, although the association between EGFR and autophagy remains unelucidated.

Mitochondrial detection of EGFR was first reported by Boerner et al (18). That study demonstrated that EGFR may translocate to the mitochondria through interaction with cytochrome $c$ oxidase subunit II, which may positively regulate survival pathways that contribute to the development of breast cancer. Recent studies indicated that mitochondrial EGFR and EGFRvIII, a constitutively activated variant of EGFR, may contribute to tumor resistance to anticancer therapy through the regulation of autophagy (20,30). EGFR mitochondrial translocation may be enhanced in A431 cells by rapamycin, an autophagy inducer. Conversely, autophagy inhibition by 3-methyladenine (an inhibitor of autophagy) or Beclin 1 knockdown by small interfering RNA (siRNA) leads to a reduction of the rapamycin-induced mitochondrial import of EGFR (20). Cao et al (31) demonstrated that tumor cells exhibiting mitochondrial EGFRvIII accumulation were highly resistant to Iressa-mediated growth inhibition and mitochondrial accumulation of EGFR as well as EGFRvIII, contributes to cancer drug resistance. Thus, under certain unfavorable and harsh microenvironment conditions, EGFR mitochondrial translocation modulated by autophagy may be critical for cell survival in certain cancer cell types.

Several target genes and microRNAs (miRNAs) are also involved in the regulation of EGFR and autophagy (Fig. 2). A knockout of the tumor susceptibility gene 101 (Tsg101), an essential requirement for cell cycle progression and cell viability, leads to decreased expression of EGFR and induction of autophagy prior to cell death, which suggests that Tsg101 knockout cells may utilize autophagy as an ultimate survival mechanism prior to their death (32). The EGFR antibody cetuximab has been proven to induce autophagy to protect cancer cells against apoptosis by downregulating HIF-1 $\alpha$ and Bcl-2 and activating the Beclin 1/hVps34 complex (33). In addition, two miRNAs, miR-221 and miR-222, were recently identified as downstream targets of the EGFR/Ras/Raf/MEK pathway, which are involved in the acquisition of resistance to antineoplastic therapies (34). However, the molecular mechanisms underlying the antitumor activity of these genes and miRNAs have not been fully elucidated.

\section{Autophagy inhibition may be a promising therapeutic strategy for enhancing the effects of EGFR-targeted ther- apy in NSCLC}

Recent studies have demonstrated that tumor resistance to anticancer therapies, including radiation therapy, chemotherapy and targeted therapies, may be increased through the upregulation of autophagy in different tumor cell lines $(35,36)$. The resistance to gefitinib and erlotinib is often mediated by acquisition of the T790M mutation, which accounts for $50 \%$ of NSCLCs $(37,38)$. Notably, EGFR T790M expression did not affect the activity of the EGFR downstream pro-survival signaling pathways. Moreover, the inhibition of signaling molecules downstream of EGFR did not enhance the sensitivity of NSCLC cells with EGFR T790M mutation to EGFR-TKIs, suggesting that there may be another route 
mediating EGFR-TKI resistance (39). A recent study demonstrated that autophagy inhibition acted synergistically with erlotinib to induce glioblastoma cell death (40). Guo et al (41) investigated the utility of autophagy-related proteins Beclin-1 and LC3 in predicting cetuximab efficacy in advanced colorectal cancer (ACRC) and observed that the patients with low Beclin-1/LC3 expression exhibited a longer progression-free survival and objective response rate compared to those with high Beclin-1 expression, indicating that autophagy may reduce cetuximab efficacy in patients with ACRC. Our laboratory recently reported that autophagy, as a cytoprotective response, may be activated by EGFR-TKIs in lung cancer cells and autophagy inhibition by chloroquine (CQ) and siRNAs targeting ATG5 and ATG7 enhanced the cytotoxicity effect of the EGFR-TKIs gefitinib and erlotinib (13). These results indicate that the combination of autophagy inhibitors and EGFR-TKIs may represent novel clinical strategies for enhancing the cytotoxic effect of EGFR-TKIs.

Several autophagy inhibitors, such as CQ and hydroxychloroquine (HCQ) have been investigated in preclinical studies or clinical trials. CQ and HCQ are antimalarial drugs that recently attracted attention as potential anticancer agents and chemosensitizers, when used in combination with anticancer drugs (42-45). In order to assess the efficacy of the combination of HCQ with conventional chemotherapeutics, three clinical trials (NCT00809237, NCT01649947, NCT00977470) are currently being launched. A phase I study on advanced NSCL patients with prior clinical benefit from EGFR-TKIs investigated the safety, maximum tolerated dose, clinical response and pharmacokinetics of HCQ, with and without erlotinib. As a result, HCQ, with or without erlotinib, was shown to be safe and the recommended phase II dose for HCQ with $150 \mathrm{mg}$ erlotinib is $1,000 \mathrm{mg}$ daily (46). Although this study did not elaborate on the difference in survival, the safety of adding HCQ to erlotinib was established. However, the efficacy of the combination of HCQ with EGFR-TKIs requires further investigation.

\section{Issues and perspectives}

NSCLC cells with EGFR mutations are sensitive to the small-molecule EGFR-TKIs gefitinib and erlotinib, although acquired resistance eventually develops. EGFR/EGFRvIII on the cell surface and mitochondria may transmit pro-survival and antiapoptotic signals, such as PI3K/AKT/mTOR. All these signals exert a strong stimulatory effect on autophagy. The cytoplasmic EGFR may modulate autophagy, whereas EGFR mitochondrial translocation may be increased by autophagy.

Autophagy, as a lysosomal degradation process, is involved in the development of cancer cell resistance to certain treatments (37). In addition to the mutations in the EGFR exons, recent studies $(24,38)$ have demonstrated that autophagy may be another probable route for the mediation of EGFR-TKI resistance. Although it has been previously indicated that combining EGFR-TKIs with autophagy-inducing drugs enhances the cytotoxicity of gefitinib or erlotinib through stimulating autophagic cell death, more evidence supports the role of autophagy as a survival pathway to protect tumor cells against anticancer therapy $(47,48)$. Furthermore, the inhibition of autophagy may enhance the sensitivity of cancer cells to various cancer therapies, including chemotherapy, radiotherapy or certain targeted therapies (49).

Our laboratory reported that autophagy, as a cytoprotective response, may be activated by EGFR-TKIs in lung cancer cells, which may be associated with acquired EGFR-TKIs resistance (13). Consistent with this hypothesis, Eimer et al (40) reported that inhibition of autophagy induced a marked increase in cell death induced by erlotinib. Based on that finding, the combination of autophagy inhibitors with EGFR-TKIs is attracting increasing attention in cancer therapy. However, the mechanism through which EGFR-TKIs induce autophagy has not been elucidated. One or more pathways should be focused on to elucidate the reason for autophagy in EGFR-TKI-resistant cells. The understanding of the functional association of autophagy with EGFR-TKI resistance may provide a promising therapeutic strategy to circumvent resistance and enhance the effects of EGFR-TKIs in patients with NSCLC.

\section{Acknowledgements}

This study was supported by grants from National Natural Science Foundation of China (Grant Nos. 81301891, 81272593, 81071651 and 81071963) and Zhejiang Provincial Natural Science Foundation of China (Grant No. LQ13H160008).

\section{References}

1. Siegel R, Naishadham D and Jemal A: Cancer statistics, 2012. CA Cancer J Clin 62: 10-29, 2012.

2. Shigematsu H, Lin L, Takahashi T, Nomura M, Suzuki M, Wistuba II, Fong KM, Lee H, Toyooka S, Shimizu N, et al: Clinical and biological features associated with epidermal growth factor receptor gene mutations in lung cancers. J Natl Cancer Inst 97: 339-346, 2005.

3. Riely GJ, Politi KA, Miller VA and Pao W: Update on epidermal growth factor receptor mutations in non-small cell lung cancer. Clin Cancer Res 12: 7232-7241, 2006.

4. Henson ES and Gibson SB: Surviving cell death through epidermal growth factor (EGF) signal transduction pathways: implications for cancer therapy. Cell Signal 18: 2089-2097, 2006.

5. Yarden Y and Shilo BZ: SnapShot: EGFR signaling pathway. Cell 131: 1018, 2007.

6. Kobayashi S, Boggon TJ, Dayaram T, Janne PA, Kocher O, Meyerson M, Johnson BE, Eck MJ, Tenen DG and Halmos B: EGFR mutation and resistance of non-small-cell lung cancer to gefitinib. N Engl J Med 352: 786-792, 2005.

7. Klionsky DJ, Abdalla FC, Abeliovich H, et al: Guidelines for the use and interpretation of assays for monitoring autophagy. Autophagy 8: 445-544, 2012.

8. Yang Z and Klionsky DJ: Eaten alive: a history of macroautophagy. Nat Cell Biol 12: 814-822, 2010.

9. Kondo Y, Kanzawa T, Sawaya R and Kondo S: The role of autophagy in cancer development and response to therapy. Nat Rev Cancer 5: 726-734, 2005.

10. Janku F, McConkey DJ, Hong DS and Kurzrock R: Autophagy as a target for anticancer therapy. Nat Rev Clin Oncol 8: 528-539, 2011.

11. Maiuri MC, Galluzzi L, Morselli E, Kepp O, Malik SA and Kroemer G: Autophagy regulation by p53. Curr Opin Cell Biol 22: 181-185, 2010.

12. Glick D, Barth S and Macleod KF: Autophagy: cellular and molecular mechanisms. J Pathol 221: 3-12, 2010.

13. Han W, Pan H, Chen Y, et al: EGFR tyrosine kinase inhibitors activate autophagy as a cytoprotective response in human lung cancer cells. PLoS One 6: e18691, 2011.

14. Sridhar SS, Seymour L and Shepherd FA: Inhibitors of epidermal-growth-factor receptors: a review of clinical research with a focus on non-small-cell lung cancer. Lancet Oncol 4: 397-406, 2003 
15. Galizia G, Lieto E, De Vita F, Orditura M, Castellano P, Troiani T, Imperatore V and Ciardiello F: Cetuximab, a chimeric human mouse anti-epidermal growth factor receptor monoclonal antibody, in the treatment of human colorectal cancer Oncogene 26: 3654-3660, 2007.

16. Vecchione L, Jacobs B, Normanno N, Ciardiello F and Tejpar S: EGFR-targeted therapy. Exp Cell Res 317: 2765-2771, 2011.

17. Lin SY, Makino K, Xia W, Matin A, Wen Y, Kwong KY, Bourguignon L and Hung MC: Nuclear localization of EGF receptor and its potential new role as a transcription factor. Nat Cell Biol 3: 802-808, 2001

18. Boerner JL, Demory ML, Silva C and Parsons SJ: Phosphorylation of Y845 on the epidermal growth factor receptor mediates binding to the mitochondrial protein cytochrome $c$ oxidase subunit II. Mol Cell Biol 24: 7059-7071, 2004.

19. Han W and Lo HW: Landscape of EGFR signaling network in human cancers: biology and therapeutic response in relation to receptor subcellular locations. Cancer Lett 318: 124-134, 2012.

20. Yue X, Song W, Zhang W, Chen L, Xi Z, Xin Z and Jiang X Mitochondrially localized EGFR is subjected to autophagic regulation and implicated in cell survival. Autophagy 4: 641-649, 2008.

21. Yarden Y and Sliwkowski MX: Untangling the ErbB signalling network. Nat Rev Mol Cell Biol 2: 127-137, 2001.

22. Ciardiello F and Tortora G: EGFR antagonists in cancer treatment. N Engl J Med 358: 1160-1174, 2008

23. Yan J, Yang H, Wang G, Sun L, Zhou Y, Guo Y, Xi Z and Jiang X: Autophagy augmented by troglitazone is independent of EGFR transactivation and correlated with AMP-activated protein kinase signaling. Autophagy 6: 67-73, 2010.

24. Cardone L, Bardelli A and Avvedimento VE: Activation of $\beta$-catenin by oncogenic PIK3CA and EGFR promotes resistance to glucose deprivation by inducing a strong antioxidant response. PLoS One 7: e37526, 2012.

25. Cao C, Lu S, Jiang Q, et al: EGFR activation confers protections against UV-induced apoptosis in cultured mouse skin dendritic cells. Cell Signal 20: 1830-1838, 2008.

26. Ghildiyal R, Dixit D and Sen E: EGFR inhibitor BIBU induces apoptosis and defective autophagy in glioma cells Mol Carcinog: Jun 29, 2012 (Epub ahead of print). doi: 10.1002/mc.21938.

27. Akhtar S, Yousif MH, Chandrasekhar B and Benter IF: Activation of EGFR/ERBB2 via pathways involving ERK1/2, P38 MAPK, AKT and FOXO enhances recovery of diabetic hearts from ischemia-reperfusion injury. PLoS One 7: e39066, 2012.

28. Chen KL, Chang WS, Cheung CH, et al: Targeting cathepsin S induces tumor cell autophagy via the EGFR-ERK signaling pathway. Cancer Lett 317: 89-98, 2012.

29. Yu Y, Fan SM, Ye YC, Tashiro S, Onodera S and Ikejima T: The tyrphostin AG1478 augments oridonin-induced A431 cell apoptosis by blockage of JNK MAPK and enhancement of oxidative stress. Free Radic Res 46: 1393-1405, 2012.

30. Dreier A, Barth S, Goswami A and Weis J: Cetuximab induces mitochondrial translocalization of EGFRvIII, but not EGFR involvement of mitochondria in tumor drug resistance? Tumour Biol 33: 85-94, 2012.

31. Cao X, Zhu H, Ali-Osman F and Lo HW: EGFR and EGFRvIII undergo stress- and EGFR kinase inhibitor-induced mitochondrial translocalization: a potential mechanism of EGFR-driven antagonism of apoptosis. Mol Cancer 10: 26, 2011

32. Morris CR, Stanton MJ, Manthey KC, Oh KB and Wagner KU: A knockout of the Tsg101 gene leads to decreased expression of ErbB receptor tyrosine kinases and induction of autophagy prior to cell death. PLoS One 7: e34308, 2012.
33. Li X and Fan Z: The epidermal growth factor receptor antibody cetuximab induces autophagy in cancer cells by downregulating HIF- $\alpha$ and Bcl-2 and activating the beclin $1 / \mathrm{hVps} 34$ complex. Cancer Res 70: 5942-5952, 2010

34. Teixeira AL, Gomes M and Medeiros R: EGFR signaling pathway and related-miRNAs in age-related diseases: the example of miR-221 and miR-222. Front Genet 3: 286, 2012.

35. Hu YL, Jahangiri A, Delay M and Aghi MK: Tumor cell autophagy as an adaptive response mediating resistance to treatments such as antiangiogenic therapy. Cancer Res 72: 4294-4299, 2012.

36. Zou Z, YuanZ,Zhang Q, et al: Aurora kinase A inhibition-induced autophagy triggers drug resistance in breast cancer cells. Autophagy 8: 1798-1810, 2012.

37. Regales L, Balak MN, Gong Y, Politi K, Sawai A, Le C, Koutcher JA, Solit DB, Rosen N, Zakowski MF and Pao W: Development of new mouse lung tumor models expressing EGFR T790M mutants associated with clinical resistance to kinase inhibitors. PLoS One 2: e810, 2007.

38. Nguyen KS, Kobayashi S and Costa DB: Acquired resistance to epidermal growth factor receptor tyrosine kinase inhibitors in non-small-cell lung cancers dependent on the epidermal growth factor receptor pathway. Clin Lung Cancer 10: 281-289, 2009

39. Moreira-Leite FF, Harrison LR, Mironov A, Roberts RA and Dive C: Inducible EGFR T790M-mediated gefitinib resistance in non-small cell lung cancer cells does not modulate sensitivity to PI103 provoked autophagy. J Thorac Oncol 5: 765-777, 2010.

40. Eimer S, Belaud-Rotureau MA, Airiau K, Jeanneteau M, Laharanne E, Veron N, Vital A, Loiseau H, Merlio JP and Belloc F: Autophagy inhibition cooperates with erlotinib to induce glioblastoma cell death. Cancer Biol Ther 11: 1017-1027, 2011.

41. Guo GF, Jiang WQ, Zhang B, Cai YC, Xu RH, Chen XX, Wang F and Xia LP: Autophagy-related proteins Beclin-1 and LC3 predict cetuximab efficacy in advanced colorectal cancer. World J Gastroenterol 17: 4779-4786, 2011.

42. Nilsson JR: Does chloroquine, an antimalarial drug, affect autophagy in Tetrahymena pyriformis? J Protozool 39: 9-16, 1992.

43. Gunja N, Roberts D, McCoubrie D, Lamberth P, Jan A, Simes DC, Hackett P and Buckley NA: Survival after massive hydroxychloroquine overdose. Anaesth Intensive Care 37: 130-133, 2009.

44. Sasaki K, Tsuno NH, Sunami E, et al: Chloroquine potentiates the anti-cancer effect of 5-fluorouracil on colon cancer cells. BMC Cancer 10: 370, 2010

45. Sasaki K, Tsuno NH, Sunami E, et al: Resistance of colon cancer to 5-fluorouracil may be overcome by combination with chloroquine, an in vivo study. Anticancer Drugs 23: 675-682, 2012.

46. Goldberg SB, Supko JG, Neal JW, et al: A phase I study of erlotinib and hydroxychloroquine in advanced non-small-cell lung cancer. J Thorac Oncol 7: 1602-1608, 2012.

47. Hu YL, Jahangiri A, Delay M and Aghi MK: Tumor cell autophagy as an adaptive response mediating resistance to treatments such as antiangiogenic therapy. Cancer Res 72: 4294-4299, 2012.

48. Li X, Lu Y, Pan T and Fan Z: Roles of autophagy in cetuximab-mediated cancer therapy against EGFR. Autophagy 6 : 1066-1077, 2010.

49. Buchser WJ, Laskow TC, Pavlik PJ, Lin HM and Lotze MT: Cell-mediated autophagy promotes cancer cell survival. Cancer Res 72: 2970-2979, 2012. 